\title{
Hospitals' readiness to implement clinical governance
}

\author{
Farbod Ebadi Fardazar ${ }^{1}$, Hossein Safari $^{2}$, Farhad Habibi ${ }^{2}$, Feyzollah Akbari Haghighi ${ }^{2}$, Aziz Rezapour ${ }^{3,4}$
}

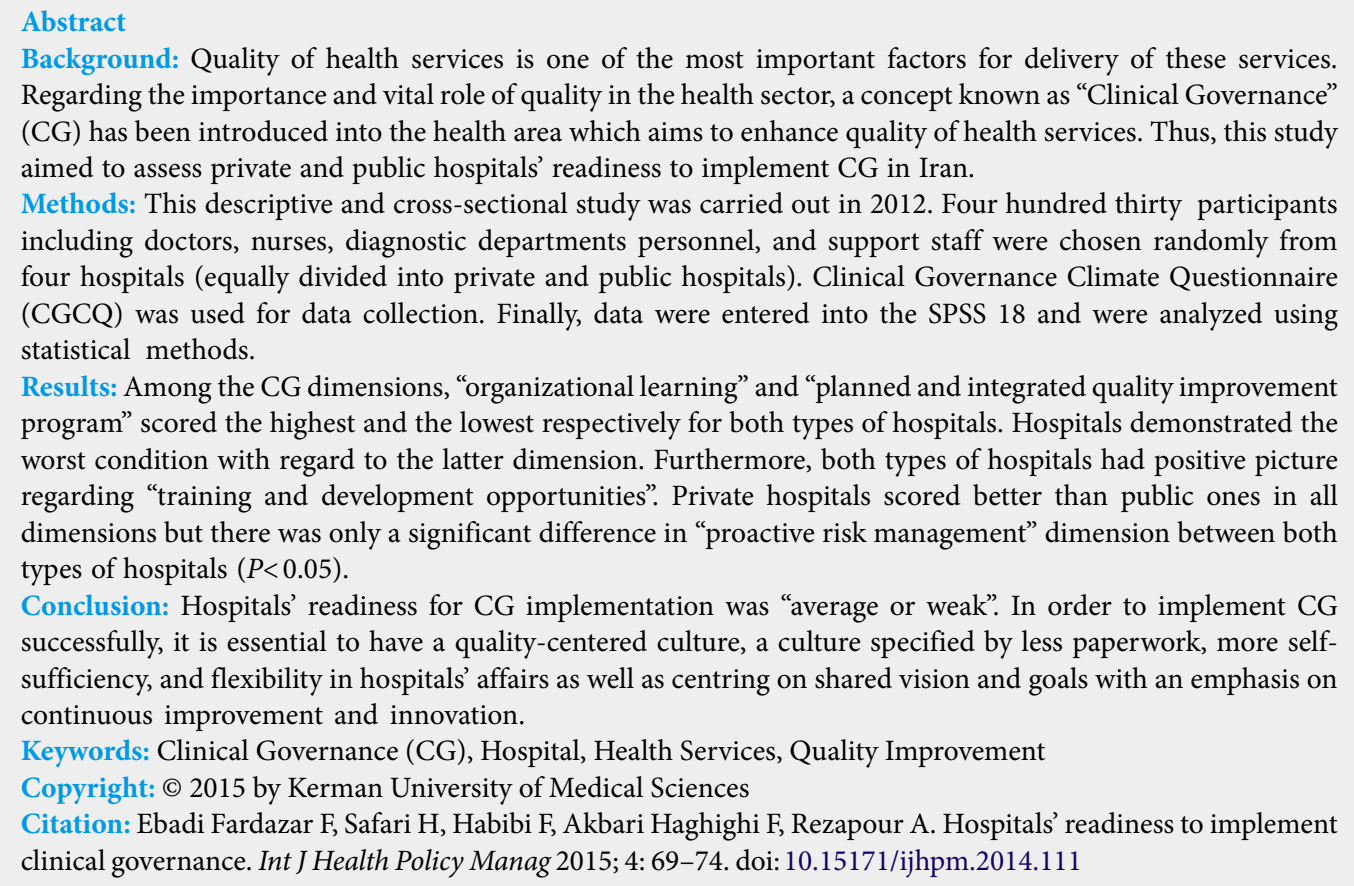
Regarding the importance and vital role of quality in the health sector, a concept known as "Clinical Governance" (CG) has been introduced into the health area which aims to enhance quality of health services. Thus, this study aimed to assess private and public hospitals' readiness to implement CG in Iran.

Methods: This descriptive and cross-sectional study was carried out in 2012. Four hundred thirty participants including doctors, nurses, diagnostic departments personnel, and support staff were chosen randomly from four hospitals (equally divided into private and public hospitals). Clinical Governance Climate Questionnaire (CGCQ) was used for data collection. Finally, data were entered into the SPSS 18 and were analyzed using statistical methods.

Results: Among the CG dimensions, "organizational learning" and "planned and integrated quality improvement program" scored the highest and the lowest respectively for both types of hospitals. Hospitals demonstrated the worst condition with regard to the latter dimension. Furthermore, both types of hospitals had positive picture regarding "training and development opportunities". Private hospitals scored better than public ones in all dimensions but there was only a significant difference in "proactive risk management" dimension between both types of hospitals $(P<0.05)$.

Conclusion: Hospitals' readiness for CG implementation was “average or weak". In order to implement CG successfully, it is essential to have a quality-centered culture, a culture specified by less paperwork, more selfsufficiency, and flexibility in hospitals' affairs as well as centring on shared vision and goals with an emphasis on continuous improvement and innovation.

Keywords: Clinical Governance (CG), Hospital, Health Services, Quality Improvement Copyright: (c) 2015 by Kerman University of Medical Sciences

Citation: Ebadi Fardazar F, Safari H, Habibi F, Akbari Haghighi F, Rezapour A. Hospitals' readiness to implement clinical governance. Int J Health Policy Manag 2015; 4: 69-74. doi: 10.15171/ijhpm.2014.111

Article History:

Received: 25 April 2014

Accepted: 27 October 2014

ePublished: 30 October 2014

\section{Key Messages}

Implications for policy makers

- Hospitals in Iran need to establish an integrated plan for quality improvement. Meanwhile, a strong committed leadership is necessary for Clinical Governance (CG) implementation to be successful.

- $\quad$ G itself does not bring about improvements. It is a team-based approach and requires commitment and efforts of all hospital personnel.

- A quality-centered culture specified by less paperwork, more self-sufficiency, and flexibility in hospitals' affairs with an emphasis on continuous improvement and innovation is essential for CG implementation.

Implications for public

Patients are the center of every healthcare system, and Clinical Governance (CG) has stepped in to take this importance into account. This study has tried to warn policy-makers about the readiness of hospitals for implementation of CG in Iran. It is the patients' right to look for quality health services and this can be brought to them by CG.

\section{Introduction}

Nowadays, in developed and most of the developing countries, governments, in general, and health sectors, in particular, create and develop organizations, entities as well as processes in order to guarantee quality improvement of health services (1-5). Surprisingly, sometimes there are differences between what policies say and what is practically done. It seems that designing a national strategy for improving quality of health services is not impossible but putting it into practice may face with many challenges (5). Moreover, quality of health services is one of the most important criteria in the health sector. It is defined as "doing the right work, for the right person, at the right time and for the first time" $(5,6)$. Considering the importance of this issue in the health sector, a concept named "Clinical Governance" (CG) has been introduced into the health sector aiming to enhance quality of health services (7). Over time, health professionals have shown more interest in CG. It is a policy that makes national health organizations 
responsible for continuous improvement of services as well as providing the highest standards in order to deliver such services to individuals (7). In other words, CG is an incentive to ensure compliance and improvement of clinical standards at environmental level and it requires participation of patients and their companions, risk prevention, clinical audit, clinical effectiveness measurement, paying attention to staff's educational needs and their occupational promotion as well as appropriate use of information $(5,8)$.

Despite the existence of many studies on CG, its concept is still not well understood and defined among health professionals (9), therefore, there is no proper framework for introduction and implementation of this initiative in the health sector. No doubt that health service providers will find themselves in a dilemma while putting CG into practice and at the time of developing policies and structures as well as using managerial and quality-oriented initiatives (9). Moreover, the issue of implementing relevant quality change initiatives becomes much more complicated and ambiguous in hospitals as important parts of the healthcare system and as key factors to the process of every health sector reform due to their strong cultural barriers related to their nature and organizational life $(9,10)$. These barriers indicate a real menace for the introduction of CG initiatives that challenge long-established culture(s), climate(s), and practices and ask for a fundamental re-focus on the way clinical quality is conceptualized and addressed (11). Therefore, any effort for making changes within the healthcare provider organizations would fail if they did not consider organizational culture (12). Although, this pays attention to different subcultures that may exist and interact within the organization, it can become much more complicated especially for hospital setting (13). CG needs massive organizational and environmental changes followed with clinical leadership and supportive culture (14). According to Haslock, introduction of CG into the health systems should be based on a systematic review of its strengths and weaknesses with an aim to use its results in daily activities as well as service delivery structures. In this policy, instead of purely creating structures, every staff is able to use the knowledge and ability in order to enhance their daily performance through which total improvement will happen in the whole policy $(5,14,15)$.

CG aims to live up to the expectations of health professionals and patients in order to provide initiatives for organizational development as well as delivering quality, sustainable and patient-centered health services. In addition, it prepares a framework which includes clinical guidelines both for health providers (doctors, nurses etc.) and health managers in order to help them address clinical issues (9). Therefore, it could be said that CG, through making a real partnership between patients, professionals and healthcare providers, offers a chance to hospitals to re-set and find their ways to move out of a constant condition towards a new and "patient-centered" culture. A culture in which there is a dynamic learning and development and questions are raised in the spirit of "trustworthy curiosity" (16-18). Concept of CG demands us to consider three following dimensions:

- Accountability; responsibility for the delivery of safe and effective care

- Quality assurance of clinical services delivered to patients

- Innovation; includes the development of new ways to practice

Evidence mainly shows the positive effects of the first dimension at the time of CG implementation (19). Successful CG implementation depends on creating a balance between three above-mentioned dimensions. According to what raised above, it is clear that for CG to be introduced fruitfully within healthcare organizations, a pivotal shift is required in their culture in order to accomplish a "cultural fit". This shifting is a more complicated issue in the hospital setting which is undoubtedly resistant to change both "structurally and culturally" $(9,10)$. Actually, hospital structures, particularly those of public ones, are hierarchical, inflexible, bureaucratic and are established in a rigid way often a long time ago (20). To sum up, hospital structures, mostly public ones, are based on a strong culture specified with features such as the "dominant orthodoxy of doctor is King" in medical practices $(13,21)$, blame and lack of frankness and participation, improper usage of information, lack of systematic learning from good practices/failures and feedback on performance routines which all are in contrast with the effective implementation of CG. So far, there has been no similar study in Iran except a few (5), because this is a newly introduced initiative in Iran. With regard to this knowledge gap, we decided to run current study in Iran. With consideration to what is mentioned above, this study was intended to assess private and public hospitals' readiness for CG implementation and to see whether they have the required bases for such implementation or not.

\section{Health system in Iran: a brief description}

Iran has augmented investment in health during the last ten years. Total expenditure on health as percentage of GDP has increased from $5.1 \%$ in 2002 to $6.7 \%$ in 2012 (Figure 1).

In spite of government spending on health, Out-of-Pocket (OOP) expenditure on health is as high as $55 \%$. It is predicted that with the introduction of "health sector evolution" plan, this rate will decrease dramatically (22).

As shown in Table 1, Iran has got four main insurance funds each of them covering a different range of population. Of course, their customers differ according to the criteria they set for coverage.

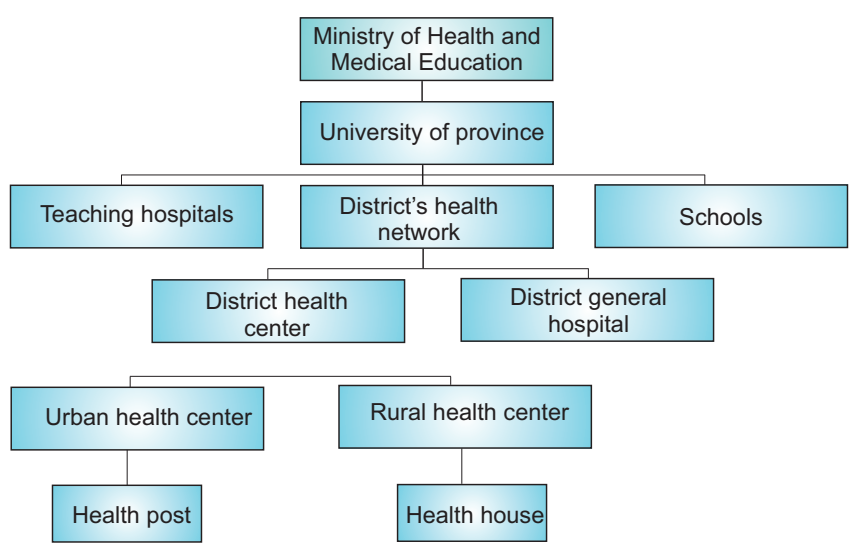

Figure 1. Health system structure in Iran 
Table 1. Health insurance system in Iran

\begin{tabular}{|c|c|c|}
\hline Main insurance funds & Coverage & Customers \\
\hline Social Security Health Insurance Organization & About 34 million & $\begin{array}{l}\text { Workers and employees in the private sector where coverage is compulsory } \\
\text { by law }\end{array}$ \\
\hline Imdad (Relief) Committee Health Insurance (ICHI) & About 1.650 .0000 & Poor citizens \\
\hline The Medical Services Insurance Organization (MSIO) & About 39 million & $\begin{array}{l}\text { Government employees, rural people, self-employed, and other sections like } \\
\text { university students, religious school students and martyr's families }\end{array}$ \\
\hline $\begin{array}{l}\text { The Armed Forces Medical Services Insurance } \\
\text { Organization (AFMSIO) }\end{array}$ & About 4 million & The armed forces and their families \\
\hline
\end{tabular}

\section{Methods}

Study design

This descriptive and cross-sectional study was set out in 2012. Two hundred fifteen participants were chosen from each type of hospitals using two-stage sampling method with $95 \%$ Confidence Interval (CI), test power of $80 \%$, and with an error less than 3 scores. Iran has 1,104 hospitals in total which 172 of them (52 private and 120 public) are in capital city of Tehran. Public hospitals are controlled and funded by the government but private ones have personal ownership. Since at the time of study CG was new for most of the hospitals and only some hospitals in the capital had implemented this system, therefore our participants were mainly chosen from hospitals in the capital. For start, because we aimed to evaluate hospitals' readiness to implement CG, so those that had implemented CG until the time of study (five private and 20 public hospitals) were excluded. The remaining 147 hospitals entered the study. A stratified sampling was carried out in the first step resulting in two public and private strata. Next, two hospitals were chosen from each stratum (overall 4 hospitals) using simple random sampling. Finally, medical, nursing, diagnostic departments, and administrative personnel were selected from each type of hospitals. Participants were distributed among hospitals proportionately based on the number of workforces in the hospitals.

Data were collected using Clinical Governance Climate Questionnaire (CGCQ). The CGCQ was developed by Freeman (15) as an indicator of the internal quality improvement aspects of CG. It consists of 60 closed items which span across six dimensions: Planned and integrated quality improvement (21 items), Proactive risk management (11 items), Absence of unjust blame and punishment (9 items), Working with colleagues (6 items), Training and development (8 items), and finally, Organizational learning (5 items). The questionnaire is scored from 1 (strongly agree) to 5 (strongly disagree) in a Likert manner. In order to verify the validity of questionnaire, questions were back translated, and then they were given to healthcare management professionals in order to use their comments. For its internal consistency, a pilot study was done on 15 participants in a four-week period using test-retest method. Cronbach's alpha was computed for each of the six dimensions.

\section{Study procedure}

Researcher referred to the hospitals and explained the research objectives for the participants and they filled informed consent forms and participated voluntarily in the study. Questionnaires were distributed "in person". With consideration to hospital type, 242 and 153 questionnaires were gathered from public and private hospitals respectively. In order to assess hospitals' readiness for CG implementation, we recorded total scores and then we changed scores into percentage. In other words, we compared scale of scores with 0 to 100 scaling and their mean (i.e. 50) was chosen as a cut point in order to determine hospitals' readiness for implementing CG dimensions. Less than 50 shows that the hospitals' readiness to implement CG is "weak to moderate" and over 50 indicates that hospitals are highly ready to implement CG.

\section{Statistical analysis}

Data were entered into the SPSS 18 and were analyzed using Chi-square, T-test, and ANOVA. Ultimately, those dimensions that gained scores higher than 50 would be considered as strengths of the hospital. On the other hand, those that acquired scores less than 50 are dimensions in need of improvement.

\section{Results}

Findings related to the demographic characteristic of participants With consideration to demographic variables, most of the participants aged $<30$ and $30-40$ years old. The least and the highest ages of participants were 20 and 68 years respectively. With regard to the gender, $27.2 \%$ of participants were males and the rest were females. Mean of work experience of the participants was $10 \pm 7.3$ years. Of 430 distributed questionnaires, 395 were filled completely by participants (response rate: $91.8 \%$ ). With regard to the type of hospitals, $61.3 \%$ of participants were from public and the rest worked in private hospitals at the time of the study. The details of demographic characteristics of the respondents are presented in Table 2.

Cronbach's alpha was computed for each dimension in the context of Iran, and the results indicated internal consistency of $0.9,0.8,0.8,0.8$, and 0.5 for each dimension respectively from one to six.

Findings related to the contributory dimensions in clinical governance implementation

According to the findings, among the CG dimensions, "organizational learning" and "planned and integrated quality improvement program" scored the highest and the lowest respectively for both types of hospitals. The average score of public and private hospitals regarding the former dimension was respectively 48.8 and 54.6 out of 100 . Furthermore, this score for the latter dimension was 13.6 and 17 for public and private hospitals in order. More information on readiness of 
Table 2. Socio-demographic characteristics of participants

\begin{tabular}{lll}
\hline Variables & & Number (\%) \\
\hline \multirow{3}{*}{ Age (year) } & $<30$ & $150(39.7)$ \\
& $30-40$ & $147(38.9)$ \\
& $>40$ & $81(21.4)$ \\
Profession & Nurse & $169(43.1)$ \\
& Para-clinical & $102(26.0)$ \\
& Support staff & $74(18.9)$ \\
Work experience (year) & Physician & $47(12.0)$ \\
& $1-10$ & $227(60.7)$ \\
Gender & $11-20$ & $115(30.7)$ \\
& $>20$ & $32(8.6)$ \\
Hospital & Male & $107(27.2)$ \\
& Female & $287(72.8)$ \\
\hline
\end{tabular}

private and public hospitals for implementing CG is presented in Table 3.

As shown in Table 3, the two private hospitals indicated a positive picture in all six examined CG dimensions but this difference was only statistically significant for "proactive risk management" dimension $(P=0.00)$. Moreover, as Figure 2 indicates, private and public hospitals' readiness was evaluated as "average or weak" to implement CG but private hospitals had a much better condition in comparison to public ones.

\section{Discussion}

This study is among the first studies conducted in Iran in order to measure hospitals' readiness for CG implementation. Results of present study showed that the Persian version of CGCQ could be considered as a fairly reliable dimension of CG climate for Iran's hospitals.

According to our study's results, public hospitals' readiness for implementing the first dimension of CG was lower than private ones. However, results of a study carried out by National Health Service (NHS) (23) showed that $70.1 \%$ of studied organizations thought that they were ready to implement this dimension of CG. Of course, it is not possible to have a direct comparison between results of our study with NHS due to the differences in methods, study structure, and scoring procedure. The key features for successful implementation of first dimension identified in the present paper are not consistent with the findings provided by other researches $(9,24)$. In this study, hospitals demonstrated the worst condition with regard to "planned and integrated program" dimension. Private hospitals signposted a positive picture compared to public ones as mentioned by Karassavidou et al. (9). The main reason for superiority of private hospitals over public ones was the supports given by high-order managers to this dimension.

In NHS study, 67\% of responsible officers reported that they are ready to implement CG with consideration to "proactive risk management" dimension. This finding is in consistent with some of other studies $(6,9,25,26)$. In these studies, preceding dimension has been considered as the most effective factor for quality improvement and facilitation of CG implementation. This was the only factor in which private hospitals demonstrated statistically a significant picture in comparison to public ones. It seems that this issue is because of mandating risk management program activities by legislatures, regulators, and insurers as well as increasing exposure to malpractice claims that impose costs on private hospitals.

Considering the third dimension, we had private hospitals scoring better than public ones. Based on NHS study, $41.6 \%$ of participants declared their readiness to implement CG in this dimension. As Moran and Luthans pointed out, lack of punishment and blame culture and provision of teaching and development chances as well as continuous learning can facilitate implementation of CG $(27,28)$. Although, these dimensions received higher score in our study in comparison to others, these scores, especially for public hospitals, were not satisfying. Meyer and Smith also stated that "Training and development (T\&D) opportunities for learning" is the most effective factor for commitment to reach goals, trust, and respect among organization members.

Table 3. Readiness of private and public hospitals to implement CG dimensions

\begin{tabular}{|c|c|c|c|c|c|c|c|}
\hline \multirow{3}{*}{ CG dimensions } & & \multicolumn{4}{|c|}{ Hospital } & \multirow{3}{*}{$\begin{array}{l}\text { Total } \\
(\%)\end{array}$} & \multirow{3}{*}{ Test results } \\
\hline & & \multicolumn{2}{|c|}{ Public } & \multicolumn{2}{|c|}{ Private } & & \\
\hline & & Number & $\%$ & Number & $\%$ & & \\
\hline \multirow{2}{*}{$\begin{array}{l}\text { Planned and integrated quality } \\
\text { improvement program }\end{array}$} & $<50$ & 209 & 86.4 & 127 & 83.0 & $336(85.0)$ & $\begin{array}{c}x^{2}=0.831 \\
d f=1\end{array}$ \\
\hline & $>50$ & 33 & 13.6 & 26 & 17.0 & $59(15.0)$ & $P=0.36$ \\
\hline \multirow{2}{*}{ Proactive risk management } & $<50$ & 177 & 73.1 & 84 & 54.9 & $261(66.0)$ & $\chi^{2}=13.910$ \\
\hline & $>50$ & 65 & 26.9 & 69 & 45.1 & $134(66.0)$ & $\begin{array}{c}d t=1 \\
P=0.00\end{array}$ \\
\hline \multirow{2}{*}{ Absence of unjust blame and punishment } & $<50$ & 163 & 67.4 & 101 & 66.4 & $265(67.0)$ & $\begin{array}{c}x^{2}=0.076 \\
d f=1\end{array}$ \\
\hline & $>50$ & 79 & 32.6 & 51 & 33.6 & $130(33.0)$ & $P=0.78$ \\
\hline \multirow{2}{*}{ Working with colleagues } & $<50$ & 196 & 81.0 & 121 & 79.6 & $317(80.0)$ & $\chi^{2}=0.094$ \\
\hline & $>50$ & 46 & 19.0 & 31 & 20.4 & 78 (20.0) & $\begin{array}{c}\mathrm{dT}=1 \\
P=0.75\end{array}$ \\
\hline Training and development opportunities & $<50$ & 139 & 57.4 & 81 & 53.3 & $220(55.6)$ & $\chi^{2}=0.768$ \\
\hline \multirow{2}{*}{ Organizational learning } & $<50$ & 124 & 51.2 & 69 & 45.4 & $193(48.8)$ & $x^{2}=1.415$ \\
\hline & $>50$ & 118 & 48.8 & 83 & 54.6 & $202(51.2)$ & $P=0.23$ \\
\hline
\end{tabular}

$\mathrm{CG}=$ Clinical Governance 


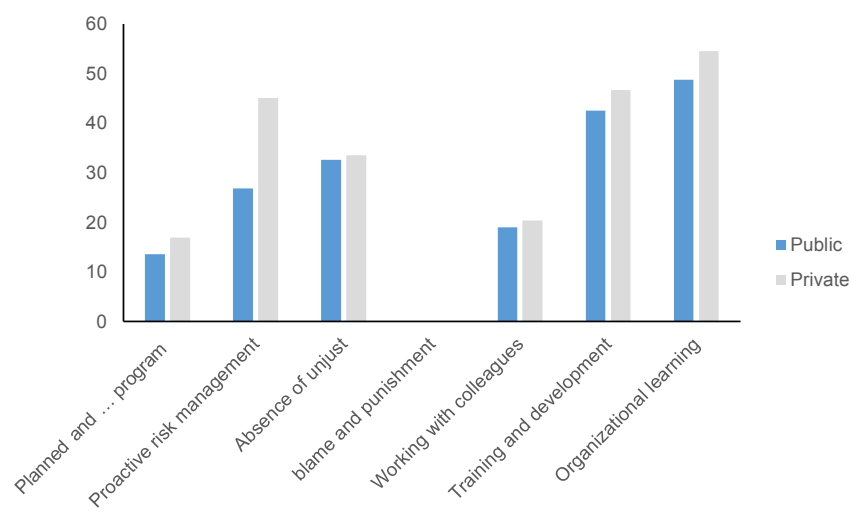

Figure 2. The average score of private and public hospitals strongly ready for $\mathrm{CG}$ implementation

In case of "working with colleagues," private hospitals again indicated a better performance than public ones but this distinction was weak in comparison to NHS standard (67\%). According to a study done by Haslock (14) participation between staff plays a very important role in successful implementation of CG. Our study, on the contrary, revealed that both types of hospitals received lower scores in this regard. In addition, McSherry et al. believed that lack of involvement among hospital personnel neutralizes the role that CG can play in patients' health (29). CG is not designed for individual affairs; it needs teams and individuals to work together. Healthcare and CG are team-based and are dependent on direct and indirect support of individuals as well as different hospital groups (30).

Both kinds of hospitals had positive picture regarding "training and development opportunities", and they scored better in comparison to NHS standard. Private hospitals, once more, indicated a relative superiority on public ones. Studies have shown that improving a culture that can enforce incentives and infrastructures for professionals lies in the effective communication and information channels within organization. This will not happen unless works are done by skillful, knowledgeable, and decent personnel $(31,32)$. Results were promising about the final dimension of CG. Both types of hospitals revealed a good condition regarding organizational learning. There seen no significant differences between hospitals in this dimension $(P>0.05)$. Other studies have also found that the existence of a proactive and learning culture within hospitals has the highest correlation with successful CG implementation $(25,33,34)$.

Overall, studied hospitals were evaluated "weak to average" for CG implementation. Private hospitals had better conditions in all dimensions than public ones, but this distinction was only statistically significant for the second dimension $(P<0.05)$. Table 3 shows the importance of CG dimensions in both groups of hospitals. Organizational learning received the highest rank in both hospitals which it gives support to some similar studies stressing this point $(35,36)$.

The effect of hospital legal status on implementation of clinical governance

T-test was used to evaluate whether hospital's legal status can affect perceptions and readiness towards CG or not. Indeed, as seen in Table 3, the two public hospitals indicated a more negative condition in all six examined CG dimensions in comparison to private ones. This finding was also supported by another study (9). This unique evidence marks the need for a new, quality-oriented culture characterized by less bureaucracy, more autonomy, and flexibility in hospital's functioning, shared vision and goals as well as concentration on continuous improvement and innovation. A culture which as it is widely accepted would positively affect the implementation of CG in the public hospitals. Further studies should focus on the way that CG can be implemented successfully within hospitals as well as the effect of holding educational classes for personnel in order to pave the way for better implementation of CG.

\section{Conclusion}

In the end, it should be noted that public hospitals' perspectives in terms of development and implementation of CG dimensions are clearly dependent on the sincere interest and commitment of the policy-makers. Indeed, it is irrational to expect that by providing a governmental framework or/ and a huge investment in healthcare systems' improvement mechanisms, the healthcare system will adapt itself automatically to the conditions (37). We need fundamental changes with an emphasis on shared vision, goals and values, inspiring leadership, autonomy, flexibility in structures, personnel employment, infrastructure, reasonable wages, supportive working conditions and practices. Through these items, it could be possible to overcome barriers to achieve CG goals.

\section{Major recommendations}

The main weakness of studied hospitals was in establishment of a planned and integrated program for quality improvements; therefore, following recommendations are given accordingly:

1. Improving senior management supports as agents to make relevant changes;

2. Providing opportunities for staff to participate in all stages of quality improvement programs;

3. Creating a blame-free atmosphere for making a "learning from mistakes" culture;

4. Identifying educational needs of staff on planned and integrated program for quality improvements.

Finally, it is worth bearing in mind that CG is in its initial steps in Iran and it needs collaboration of all health sector authorities and staff. Meanwhile, readers should notice that there are other methods for assessing CG development such as the Clinical Governance Development Index (CGDI). The reason for choosing CGCQ in this study was that it contained a broader range of areas for assessment.

\section{Acknowledgements}

This article is resulted from an M.Sc. thesis in 2012 at Tehran University of Medical Sciences (TUMS). We would like to acknowledge all respondents. 
Ethical issues

Ethical issues (including plagiarism, informed consent, misconduct, data fabrication and/or falsification, double publication and/or submission, redundancy, etc.) have been completely observed by the authors. By the way, our study was approved by the ethics committee of Tehran University of Medical Sciences (TUMS).

\section{Competing interests}

The authors declare that they have no competing interests.

\section{Authors' contributions}

All authors have been contributed equally.

\section{Authors' affiliations}

${ }^{1}$ Hospital Management Research Center, Iran University of Medical Sciences, Tehran, Iran. ${ }^{2}$ Department of Health Management and Economics, Health School, Tehran University of Medical Sciences, Tehran, Iran. ${ }^{3}$ Department of Health Economics, School of Health Management and Information Science, Iran University of Medical Sciences, Tehran, Iran. ${ }^{4}$ Health Management and Economics Research Center, Iran University of Medical Sciences, Tehran, Iran.

\section{References}

1. Silimperi DR, Franco LM, van Zanten TV, Macaulay C. A framework for institutionalizing quality assurance. Int J Qual Health Care 2002; 14: 67-73. doi: 10.1093/intqhc/14.suppl_1.67

2. Power M. The audit society-Second thoughts. International Journal of Auditing 2000; 4: 111-9. doi: 10.1111/1099-1123.00306

3. Hood C, James O, Scott C. Regulation of government: has it increased, is it increasing, should it be diminished? Public Adm 2000; 78: 283-304. doi: 10.1111/1467-9299.00206

4. O'neill O. A question of trust: The BBC Reith Lectures 2002. Cambridge: Cambridge University Press; 2002.

5. Mojtaba S. Designing and implementing clinical governance in pilot hospitals. Tehran university of medical sciences; 2006. [In Persian]

6. Scally G, Donaldson LJ. Clinical governance and the drive for quality improvement in the new NHS in England. BMJ 1998; 317 : 61-5. doi: 10.1136/bmj.317.7150.61

7. Donaldson LJ, Gray J. Clinical governance: a quality duty for health organisations. Qual Health Care 1998; 7: S37-44.

8. Kassirer JP. The quality of care and the quality of measuring it. $N$ EnglJ Med 1993; 329: 1263. doi: 10.1056/nejm199310213291710

9. Karassavidou E, Glaveli N, Zafiropoulos K. Assessing hospitals' readiness for clinical governance quality initiatives through organisational climate. J Health Organ Manag 2011; 25: 214-40.

10. Bahrami MA, Sabahi AA, Montazeralfaraj R, Shamsi F, Entezarian S. Hospitals' Readiness for Clinical Governance Implementation in Educational Hospitals of Yazd, Iran. Electronic Physician 2014; 6: 794-800.

11. Konteh FH, Mannion R, Davies HT. Clinical governance views on culture and quality improvement. Clinical Governance: An International Journal 2008; 13: 200-7. doi: 10.1108/14777270810892610

12. Ekvall G. Climate, structure and innovativeness of organizations: A theoretical framework and an experiment. Stockholm: FArådet, the Swedish Council for Management and Organizational Behaviour; 1983.

13. Morgan PI, Ogbonna E. Subcultural dynamics in transformation: a multi-perspective study of healthcare professionals. Hum Relat 2008; 61: 39-65. doi: 10.1177/0018726707085945

14. Haslock I. Introducing clinical governance in an acute trust. Hosp Med 1999; 60: 744-7. doi: 10.12968/hosp.1999.60.10.1220

15. Freeman T. Measuring progress in clinical governance: assessing the reliability and validity of the Clinical Governance Climate Questionnaire. Health Serv Manag Res 2003; 16: 23450. doi: 10.1258/095148403322488937

16. Nicholls S, Cullen R, O'Neill S, Halligan A. Clinical governance: its origins and its foundations. British Journal of Clinical Governance 2000; 5: 172-8. doi: 10.1108/14777270010734055

17. Rose RC, Uli J, Abdul M, Ng KL. Hospital service quality: a managerial challenge. Int $J$ Health Care Qual Assur 2004; 17: 146-59.

18. Halligan A. Clinical governance: assuring the sacred duty of trust to patients. Clinical Governance: An International Journal 2006; 11: 5-7. doi: 10.1108/14777270610646967

19. Peak M, Burke R, Ryan S, Wratten K, Turnock R, Vellenoweth C. Clinical governance-the turn of continuous improvement? Clinical Governance: An International Journal 2005; 10: 98-105. doi: 10.1108/14777270510594272

20. Preker AS, Harding A. Innovations in health service delivery: the corporatization of public hospitals. Washington, DC: World Bank Publications; 2003.

21. van Zwanenberg $T$. Clinical governance in primary care: from blind-eye to no-blame culture in one short leap? British Journal of Clinical Governance 2001; 6: 83-6. doi: 10.1108/14664100110815414

22. Mehrdad R. Health system in Iran. JMAJ 2009; 52: 69-73.

23. National Health Service (NHS). A review of integrated clinical governance in the context of medical revalidation. Britain Revalidation support team; 2011.

24. Freeman $\mathrm{T}$, Walshe $\mathrm{K}$. Achieving progress through clinical governance? A national study of health care managers' perceptions in the NHS in England. Qual Saf Health Care 2004; 13: 335-43. doi: 10.1136/qhc.13.5.335

25. Som CV. Clinical governance: a fresh look at its definition. Clinical Governance: An International Journal 2004; 9: 87-90. doi: $10.1108 / 14777270410536358$

26. Warwick C. The new NHS: modern dependable. British Journal of Midwifery 1998; 6: 265. doi: 10.12968/bjom.1998.6.4.265

27. Moran R. "There's no time to learn"... like the present. Clinical Governance: An International Journal 2003; 8: 46-56. doi: 10.1108/14777270310460010

28. Luthans KW, Lebsack SA, Lebsack RR. Positivity in healthcare: relation of optimism to performance. J Health Organ Manag 2008; 22: 178-88. doi: 10.1108/14777260810876330

29. McSherry R, Pearce P. Clinical governance. Chichester: John Wiley \& Sons; 2010.

30. McSherry R. Factors influencing collaborative working. In: McSherry R, Warr J, editors. Implementing Excellence In Your Health Care Organization: Managing, Leading And Collaborating: managing, leading and collaborating. Maidenhead: Open University Press; 2010.

31. McSherry R, Warr J. An introduction to excellence in practice development in health and social care. Maidenhead: McGrawHill International; 2008.

32. McCormack B, Manley K. Evaluating Practice Developments. In: McCormack B, Manley K, Garbett R, editors. Practice Development in Nursing. Oxford: Blackwell Publishing Ltd; 2004. doi: $10.1002 / 9780470698884 . c h 5$

33. Som CV. Exploring the human resource implications of clinical governance. Health Policy 2007; 80: 281-96. doi: 10.1016/j. healthpol.2006.03.010

34. Manley K. Transformational culture: A culture of effectiveness. In: McCormack B, Manley K, Garbett R, editors. Practice Development in Nursing. Oxford: Blackwell Publishing Ltd; 2004. doi: 10.1002/9780470698884.ch4

35. Specchia ML, La Torre G, Siliquini R, Capizzi S, Valerio L, Nardella $\mathrm{P}$, et al. OPTIGOV-A new methodology for evaluating Clinical Governance implementation by health providers. BMC Health Serv Res 2010; 10: 174. doi: 10.1186/1472-6963-10-174

36. Wilkinson JE, Rushmer RK, Davies HT. Clinical governance and the learning organization. J Nurs Manag 2004; 12: 105-13. doi: 10.1046/j.1365-2834.2003.00435.x

37. Storey J, Buchanan D. Healthcare governance and organizational barriers to learning from mistakes. J Health Organ Manag 2008; 22: 642-51. doi: 10.1108/14777260810916605 